of QoL domains need to be examined further. Identifying predictors of QoL could promote prevention and treatment plans for supporting adaptive lifestyle of adolescents.

\section{P603 UROPATHOGEN PROFILE IN THE PAEDIATRIC POPULATION - A COMPARATIVE STUDY BETWEEN TWO GEOGRAPHICALLY DISTINCT REGIONS IN IRELAND}

${ }^{1}$ Samy AA Allawendy*, ${ }^{2}$ Mahmoud Farhan, ${ }^{1}$ Alessandra Biagini, ${ }^{2}$ Abdullah Riaz, ${ }^{1}$ Ana Isabel Rueda-Benito, ${ }^{1}$ Karen Logan, ${ }^{2}$ Briain McDonald, ${ }^{1}$ Hilary Greaney, ${ }^{1}$ Ghia Harrison, ${ }^{2}$ Alan Finan. 'Sligo University Hospital, Sligo, Ireland; ${ }^{2}$ Cavan General Hospital, Cavan, Ireland

10.1136/archdischild-2019-epa.935

Background Urinary tract infection (UTI) is one of the most common bacterial infections among children. The high level of antimicrobial resistance in uropathogens worldwide is a cause for real concern. Currently empirical first line antibiotics used for UTI in children are similar in most hospitals. There is limited data in relation to geographical variation in uropathogen prevalence and their antimicrobial sensitivity. This study aims to evaluate and compare the prevalence and resistance pattern of UTI pathogens in two geographically distinct areas in Ireland.

Methods Paediatric patients admitted with an uncomplicated laboratory confirmed UTI at Sligo University Hospital and Cavan General Hospital between January 2017 and December 2018 were reviewed; Patients with structurally or neurologically complicated urinary tracts were excluded from this study. Antimicrobial susceptibility to Amox-clavulanate, Amoxycillin, Ceftazidime Fosfomycin, Gentamicin, Nitrofurantoin, and Trimethoprim was determined for urinary isolates.

Results Profiles of organisms and antimicrobial sensitivities in Sligo and Cavan patients were broadly similar but with some differences., Escherichia coli was the most prevalent pathogen contributing to UTIs representing $80.22 \%$ and $87.34 \%$ of isolates in Sligo and Cavan respectively. Highest rates of resistance were noted to Amoxycillin $(49.45 \%$ and $62.03 \%$ respectively) and co-Amox-clavulanate (42.86\% and $41.77 \%)$. Highest rates of antimicrobial sensitivity for isolates from Sligo were for Nitrofurantoin (91.2\%), followed by Fosfomycin (90.11\%) while in Cavan, they were for Gentamicin (96.2\%) followed by Nitrofurantoin and Ceftazidime (92.4\% both).

Conclusions High resistance was observed to Amoxycillin and co- Amox-clavulanate which are commonly used as empirical treatments for UTIs. It may be timely to review our local empirical antibiotic choices. The results of this small study in two local acute paediatrics services would indicate it may be useful to conduct a wider national review of uropathogen patterns and sensitivities in the paediatric population.

\section{P604 IS IT THE NEGATIVE EFFECTS OF SERUM VITAMIN DEFICIENCY OF PREGNANT AND LACTATING WOMEN ON DEVELOPMENT OF NEWBORN NEUROLOGICAL SYSTEM CAN BE AVOIDED}

Çiğdem El* ${ }^{*}$ Mehmet Emin Çelikkaya. Mustafa Kemal University, Hatay, Turkey

\subsection{6/archdischild-2019-epa.936}

Purpose Deficiency of vitaminB12 if not diagnosed and treated early in infant period where the growth and development is very rapid which are very important for the construction of
DNA/RNA and neurotransmitters, can lead to irreversible neuromotor pathologies.

Material and method Babies who were admitted with neurological complaints due to vitaminB12 deficiency between the dates of January2018-January2019 were included in this study. During the pregnancy and lactation intake of vitamin supplements, duration of breastfeeding and vitaminB12 levels were examined.

Results In our study, the mean age of 11babies was 2.6(2.13.4) months. Neurological findings; in 7babies there was swallowing disorder and in 4babies undeveloped ability to tonic neck reflex. The serum levels of vitaminB12 in babies were determined under $150 \mathrm{pg} / \mathrm{dl}$ and in all mothers was under $250 \mathrm{pg} / \mathrm{dl}$. Although they were not regular receive iron and folate supplements during pregnancy, they did not never receiving vitaminB12 supplement during pregnancy-lactation.

Discussion VitaminB12 deficiency in babies may be associated with neurological findings and that delay in the diagnosis and treatment of infants may cause permanent neurological damage. In addition, maternal deficiency is considered to be an independent risk factor for the development of fetal neural tube defect. Deficiency of vitamin B12 in pregnant and lactating mothers is the main cause of Vitamin B12 deficiency in fetus and babies.

Conclussion VitaminB12 deficiency is one of the causes of neurological retardation in babies (affect the development of the brain in the prenatal/postnatal period). In order to prevent this deficiency, healthy nutrition of the mother and taking the necessary vitamin supplements both the prenatal period and postnatal period should be provided. We think that should be given vitamin B12 routinely to all pregnant women like folic acid and iron supplementation.

\section{P605 FUNCTIONAL CONSTIPATION IN A TERTIARY HOSPITAL SETTING}

${ }^{1}$ Katarina Milošićc ${ }^{1,2}$ Mirna Natalija Aničić, ${ }^{2}$ Lana Omerza, ${ }^{1,2}$ Irena Senečić-Čala, ${ }^{1,2}$ Jurica Vuković, ${ }^{1,2}$ Duška Tješić-Drinković. 'Zagreb School of Medicine, Zagreb, Croatia; ${ }^{2}$ UHC Zagreb, Zagreb, Croatia

10.1136/archdischild-2019-epa.937

Objectives Functional constipation in children should be diagnosed in primary care, based on Rome criteria. Management in tertiary care is rarely needed. The aim of this study was to assess the frequency and structure of paediatric patients diagnosed with functional constipation in a tertiary hospital setting.

Methods This study enrolled children referred to the paediatric gastroenterologist (PG) at the UHC Zagreb from January 1st 2017 to December 31st $2017(\mathrm{~N}=1729)$. Data on patients was extracted retrospectively from clinical records. The subjects were classified in three age groups: infants and toddlers (0-3 years), children (4-10 years) and adolescents (11-18 years). Descriptive statistics and chi-square test were used, statistical significance was determined as $\mathrm{p}<0.05$

Results After appropriate evaluation, about $8 \%$ of all outpatients seen by the PG at the UHC Zagreb during year 2017 had functional constipation with or without encopresis $(7.63 \%$ or 132 patients, boys: $56,1 \%$ vs girls: 43,9\%). Half of the patients were in the age group of 4 to 10 years $(66 / 132$ or $50 \%$ ), about $1 / 3$ was 0 to 3 years old (41/132), and about $1 /$ 5 were adolescents (25/132). It was the most common functional gastrointestinal disorder (FGID) (132/328 or 40.24\%), 